\title{
Exploring a small sawtooth regime in Joint European Torus plasmas with counterinjected neutral beams
}

\author{
M. F. F. Nave \\ Associação EURATOM/IST, Centro de Fusão Nuclear, 1049-001 Lisbon, Portugal \\ H. R. Koslowski \\ Association EURATOM-FZ-Juelich, Institut fuer Plasmaphysik, Trilateral Euregio Cluster, D-52425 Juelich, \\ Germany \\ S. Coda and J. Graves \\ Association EURATOM-Confederation Suisse, EPFL SB CRPP, Station 13, 1015 Lausanne, Switzerland
}

M. Brix, R. Buttery, C. Challis, C. Giroud, M. Stamp, and P. de Vries

Euratom/UKAEA Fusion Association, Culham Science Centre, Abingdon, OX14 3DB, United Kingdom

JET-FEDA contributors

(Received 12 August 2005; accepted 5 December 2005; published online 27 January 2006)

\begin{abstract}
During a recent reversed toroidal field $\left(B_{T}\right)$ campaign at the Joint European Torus (JET), experiments were performed to investigate the effect on sawteeth of neutral beam injection (NBI)-driven toroidal plasma rotation counter to the direction of the toroidal plasma current and $B_{T}$. A power scan at constant density has permitted analytical continuation, into the reversed $B_{T}$ domain, of previous experiments with forward field and hence corotation. Earlier JET results were confirmed, indicating that counter-NBI results in sawtooth periods shorter than in the Ohmic regime. This study has demonstrated that, whereas with co-NBI the sawtooth period increases with power, with counter-NBI the sawtooth period initially decreases with power passing through a minimum at $4 \mathrm{MW}$. Clearly this trend also manifests itself in terms of the toroidal plasma rotation, for which a minimum is observed for counter-rotation frequency $\sim 3 \mathrm{kHz}$. Sawteeth smaller than Ohmic sawteeth are found to be easier to obtain with perpendicular counter-NBI, for which heating penetrates deeper into the core. The sign and magnitude of the toroidal rotation, the penetration of heating to the electrons, and the peaking of the fast-ion pressure profile in the core may all play an important role in modifying the sawtooh period. (C) 2006 American Institute of Physics.
\end{abstract}

[DOI: $10.1063 / 1.2162528$ ]

Developing techniques to control the $m=1, n=1$ sawtooth instability as a way of controlling the onset of neoclassical tearing modes (NTMs) has been an important area of research in recent Joint European Torus ${ }^{1}$ (JET) experimental campaigns. Reducing the sawtooth period was found to trigger the NTMs at higher values of normalized beta. ${ }^{2}$ In addition, the importance of sawtooth control has been demonstrated in JET high-density ELMy $H$-mode plasmas, where sawteeth play a beneficial role in the prevention of core impurity accumulation. ${ }^{3}$ In order to achieve a balance between beneficial and deleterious effects, it is important to understand how to maintain small, regularly occurring sawtooth crashes. Sawtooth stabilization leading to long sawtooth periods is a well-known phenomenon in auxiliary heated plasmas. Nevertheless, sawtooth periods shorter than observed in Ohmic regimes have been obtained at JET with ion-cyclotron radio-frequency heating ${ }^{4}$ as well as with counter-neutral beam injection (NBI) heating ${ }^{5}$ with the toroidal field $B_{T}$ reversed. Recently this small sawtooth regime with counterNBI heating has been investigated in detailed experiments, the results of which are reported here.

\footnotetext{
${ }^{a)}$ See the Appendix of J. Pamela et al., Fusion Energy 2004, Proceedings of the 20th International Conference of the IAEA, Vilamoura, Portugal.
}

JET operation with reversed $B_{T}$ is performed with the direction of the plasma current $I_{p}$ also reversed, in order to conserve the direction of impact of the field lines on the divertor target plates. JET has two neutral beam injector boxes, each equipped with positive ion neutral injectors (PINIs), which can provide up to $20 \mathrm{MW}$ of NBI power in total. ${ }^{6}$ The PINIs are grouped in tangential and nearly perpendicular banks (the latter referred here as "perpendicular" NBI). With the usual $B_{T}$ polarity the beams are injected in the direction of the plasma current, whereas with $B_{T}$ reversed NBI is in the direction counter to $I_{p}$, as illustrated in Fig. 1. Sawtooth observations in the reversed and usual $B_{T}$ machine configuration were compared in dedicated experiments in $L$-mode plasmas with matched $B_{T}$ and $I_{p}$. The shape was also carefully matched. Owing to an outward displacement of the trapped ions' banana orbits, a large wall clearance was required for the reversed $B_{T}$ operation. In addition, the effects of perpendicular versus tangential neutral injection were investigated. Results from these dedicated sawtooth experiments as well as from a wider database including many plasma configurations confirmed the earlier JET observations ${ }^{5}$ that the sawtooth period $\tau_{\mathrm{ST}}$ was reduced with reversed $B_{T}$ and $I_{p}$. The shortest observed sawtooth periods were about $1 / 3$ of the Ohmic sawtooth periods. The striking 


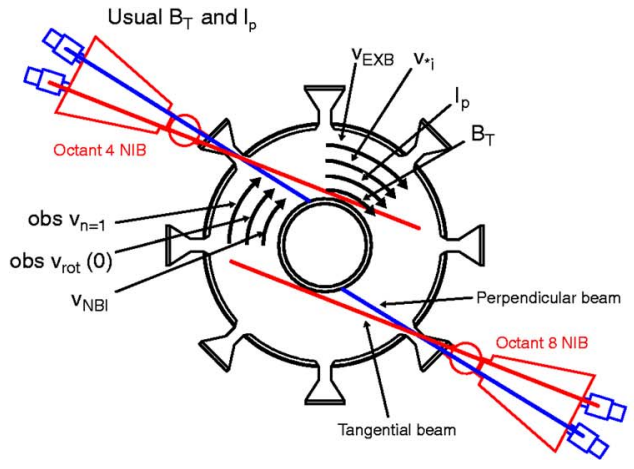

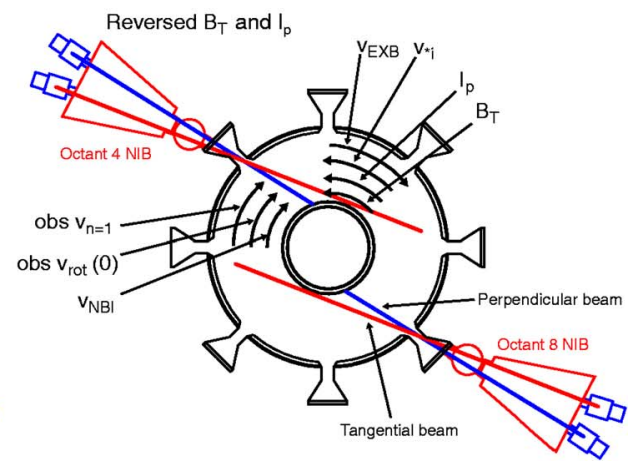

FIG. 1. (Color online) JET viewed from the top showing lines of NBI. Also indicated are directions of $I_{p}$, observed toroidal rotation at the plasma center from charge exchange measurements $\left[v_{\text {rot }}(0)\right]$, toroidal rotation from NBI momentum $\left(v_{\mathrm{NBI}}\right)$, propagation of $n=1$ sawtooth precursor from magnetic pickup coils mode analysis $\left(v_{n=1}\right)$, toroidal projections of ion diamagnetic rotation $\left(v_{* I}\right)$, and rotation from $\mathbf{E} \times \mathbf{B}$ drift $\left(v_{\mathrm{E} \times \mathrm{B}}\right)$. difference in sawtooth behavior for the same NBI power is illustrated in Fig. 2 for discharges where $P_{\mathrm{NBI}}$ was ramped up and subsequently ramped down. In order to study the effect of rotation on sawtooth stability, a power scan at constant density was performed in co- and counter-rotating plasmas. Controlled conditions at each power level were maintained for $2 \mathrm{~s}$. For this study, neutral beam injection was mainly in the perpendicular direction. The sawtooth period dependence on heating power was found to be very different when coand counter-NBI plasmas were compared [Figs. 3 and 4(a)]. With co-NBI the sawtooth period increased with power. With counter-NBI the average sawtooth period reached a minimum, $\tau_{\mathrm{ST}}{ }^{\mathrm{NBI}} \sim 27 \mathrm{~ms}$, at a NBI power $P_{\mathrm{NBI}} \sim 4 \mathrm{MW}[$ Fig. 4(a)]. For NBI powers up to $7 \mathrm{MW}$ sawtooth periods were smaller than typical Ohmic heating sawtooth periods $\left(\tau_{\mathrm{ST}}{ }^{\mathrm{Ohm}} \sim 70-80 \mathrm{~ms}\right)$.

A similar dependence of the sawtooth period on the core plasma rotation velocity, obtained from charge-exchange measurements, ${ }^{7}$ was observed [Fig. 4(b)]. With the usual $B_{T}$ and $I_{p}$ the sawtooth period increased with plasma rotation, whereas with reversed $B_{T}$ and $I_{p}$ the sawtooth period decreased to a minimum at a toroidal rotation frequency of

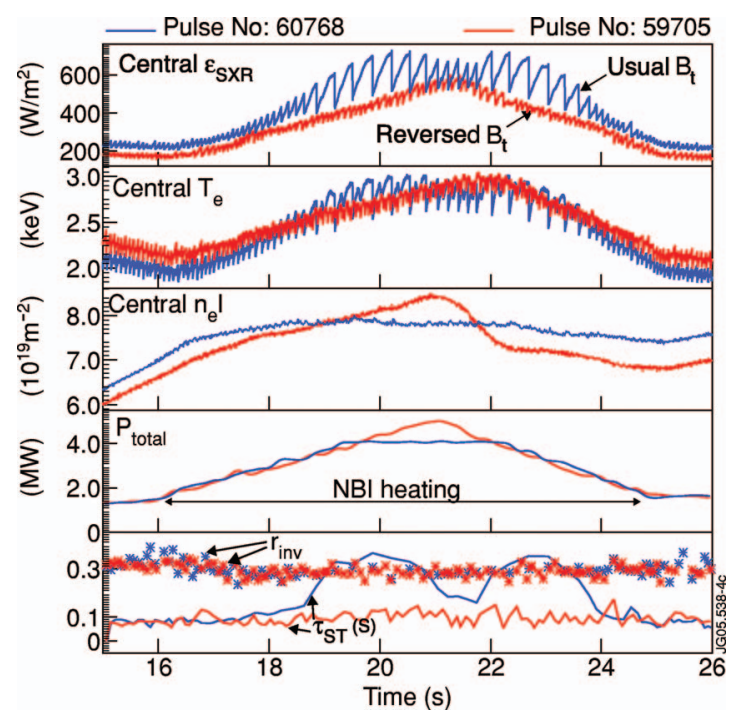

FIG. 2. (Color) A pair of discharges with normal and reversed $B_{T}$ polarities with matched shape $\left(B_{T}=2.7 \mathrm{~T}, I_{p}=2.5 \mathrm{MA}, q_{95}=3.6\right)$ using nearperpendicular NBI. The figure shows (a) SXR brightness from a central line of sight, (b) central electron temperature, (c) central density, (d) total heating power, (e) and sawtooth period (solid lines) and normalized sawtooth inversion radius on the low-field side obtained from SXR data. $f_{\text {rot }} \sim 3 \mathrm{kHz}$, measured at the sawtooth-inversion radius. With counter-NBI the toroidal rotation speed was lower than with co-NBI for similar NBI power and its direction was opposite with respect to $I_{p}$ (although the same in the laboratory frame, see Fig. 1).

A sawtooth study using reversed $B_{T}$ and coinjection in the Tokamak Experiment for Technology-Oriented Research ${ }^{8}$ (TEXTOR) has also found a minimum in the sawtooth period as a function of power (for $P_{\mathrm{NBI}} \sim 0.2 \mathrm{MW}$ ). ${ }^{9}$ Observations of sawtooth magnetohydrodynamics (MHD) precursors indicated that the minimum occurred when the observed mode rotation was zero. In JET, the sawtooth precursor always rotated in the direction of the beam injection (as indicated in Fig. 1). For counter-NBI cases the observed frequency was on average $2-3 \mathrm{kHz}$ higher than the plasma rotation frequency at the sawtooth-inversion radius. Unlike TEXTOR the minimum sawtooth period at JET does not correspond to a zero-mode frequency in the laboratory frame.

The short sawtooth periods observed earlier in reversed $B_{T}$ and $I_{p}$ plasmas were attributed to changes in the $q$ profile due to neutral beam current drive (NBCD). ${ }^{5}$ It was reported that NBCD caused a slightly reversed $q$ profile with $q_{\text {min }}$ $<1$. This has not been found in the present experiments as both the motional Stark effect (MSE) and Faraday rotation measurements indicated that the $q$ profiles are peaked. The
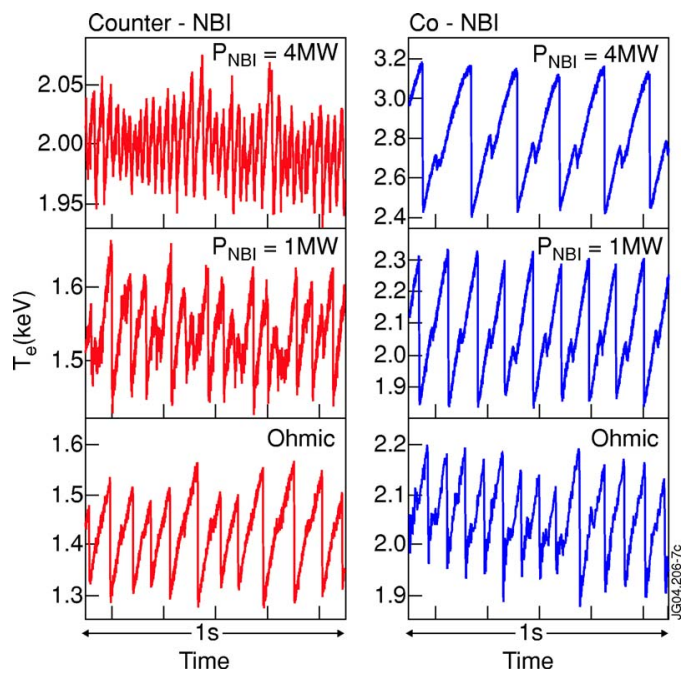

FIG. 3. (Color online) Central electron temperature traces showing sawtooth behavior for different input powers. 

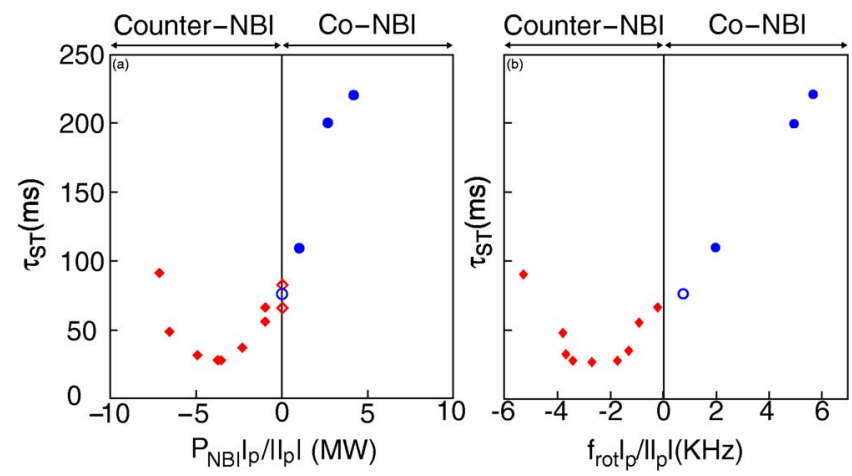

FIG. 4. (a) (Color online) Sawtooth period vs $P_{\mathrm{NBI}}$ from a power scan at constant density using mainly normal NBI (open symbols for Ohmic pulses). (b) Sawtooth period vs frequency of toroidal rotation measured at the sawtooth inversion radius. (The open symbol shows toroidal rotation frequency measured $100 \mathrm{~ms}$ after $1 \mathrm{MW}$ of co-NBI in the usual $B_{T}$. It has not been possible to determine the rotation velocity of Ohmic plasmas as the $\mathrm{Ni}^{26}$ line Doppler shift measurements were within noise level, for both the usual and reversed $B_{T}, I_{p}$ configurations.)

modification in the core current density due to NBCD, $j_{\mathrm{CD}}$, was calculated with the PENCIL code. ${ }^{10}$ It was in all cases less than $10 \%$ of the Ohmic current. Nevertheless, $j_{\mathrm{CD}}$ was larger with counterinjection and it increased with the input power. Since the NBI-driven current is in the opposite direction to the plasma current, one may expect the radius of the $q=1$ surface to decrease with increasing NBI power. As neither the $q$ profile nor the measured inversion radius changed significantly with the counter-NBI heating power applied (see Fig. 2), the effect of current drive appears to be insignificant. Moreover, in tangential counter-NBI discharges, for which the current drive was found to be much enhanced, the sawtooth period was not shorter than in similar discharges with normal injection.

While in some of the counter-NBI discharges sawtooth periods shorter than ohmic sawtooth periods were observed (as in Figs. 3 and 4), in others the sawtooth period remained close to Ohmic values (as in Fig. 2). It has been found that sawteeth smaller than Ohmic sawteeth occur for plasmas where the NBI heating power to the electrons penetrate deeply into the core. This was more often observed with perpendicular injection, although for a large core heating power density $\left[P_{e}(\psi=0.1)>1.5 \times 10^{5} \mathrm{~W} \mathrm{~m}^{-3}\right]$ sub-Ohmic sawteeth were also observed with parallel NBI. Figure 5 shows two discharges with similar ramped up counter-NBI power. Initially, when parallel NBI was used, the sawtooth period remained close to the value observed in the Ohmic phase. With the addition of perpendicular NBI (at $17.5 \mathrm{~s}$ for pulse 59706 and at $21 \mathrm{~s}$ for pulse 59707) the sawtooth period became shorter. This coincided with an increased power to the electrons. Efficient heating to the electrons in the core is expected to increase the current penetration, and thus the rate of increase of the magnetic shear with time close to the $q$ $=1$ surface. Such a process could be partially responsible for the small sawteeth observed. In the discharges shown in Fig. 5 , observation of sub-Ohmic sawtooth occurred at times when perpendicular injection was employed. These smallerthan-Ohmic sawteeth continued up to $12 \mathrm{MW}$, when equal amounts of perpendicular and parallel injections were used,

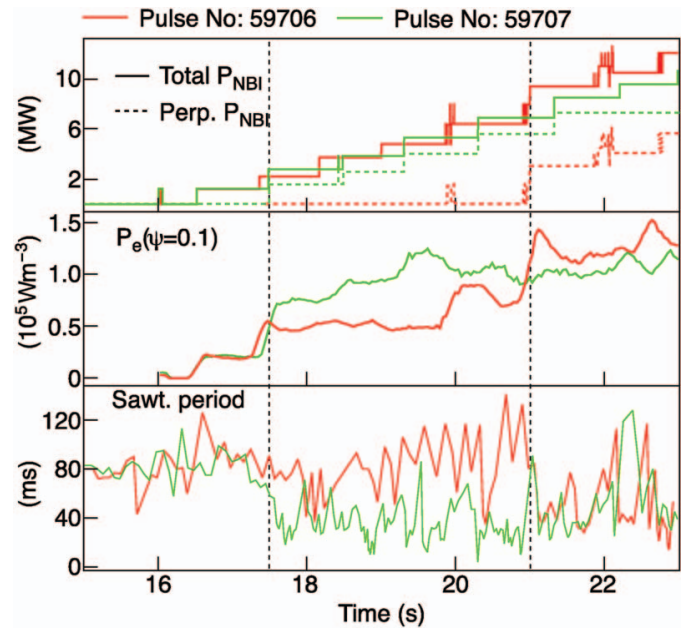

FIG. 5. (Color) Pair of discharges in the reversed $B_{T}$ and counter-NBI configuration. (a) Total and perpendicular NBI powers, (b) volume-averaged power transferred to the electrons at the flux surface $\psi=0.1$ (calculated with the PENCIL code), and (c) observed sawtooth period. The vertical lines indicate the times when perpendicular NBI was added: $t=17.5$ for pulse No. 59707 and $t=21 \mathrm{~s}$ for pulse No. 59706 .

but at $P_{\mathrm{NBI}} \sim 14 \mathrm{MW}$ sawtooth periods longer than Ohmic sawteeth were observed.

Crash criteria known as the "ITER sawtooth model" given in Ref. 11 have previously been used to model the sawtooth cycle of JET discharges. ${ }^{12}$ The increasing sawtooth period with NBI power observed in the usual $B_{T}$ configuration was found to be consistent with increasing stability from kinetic effects from fast beam ions. ${ }^{12}$ For the power scan described here, PENCIL calculations show that the fast ions' pressure increased with power for both the usual and reversed $B_{T}$ cases (Fig. 6). The lack of correlation between the observed $\tau_{\mathrm{ST}}$ and fast-ion pressure in the counter-NBI case indicates that the contribution from the fast-particle component cannot explain the observed $\tau_{\mathrm{ST}}$ dependence on $P_{\mathrm{NBI}}$. The correlation between sawtooth period and plasma rotation indicates that the effect of plasma rotation, not considered in the International Thermonuclear Experimental Reactor (ITER) sawtooth model, may be important. The kinetic effects from trapped thermal ions described in Ref. 13 are strongly stabilizing in conventional scenarios with large corotation. However, the effect of sheared flow on these kinetic effects modifies the stability threshold of the internal kink mode in such a way that the critical beta for instability
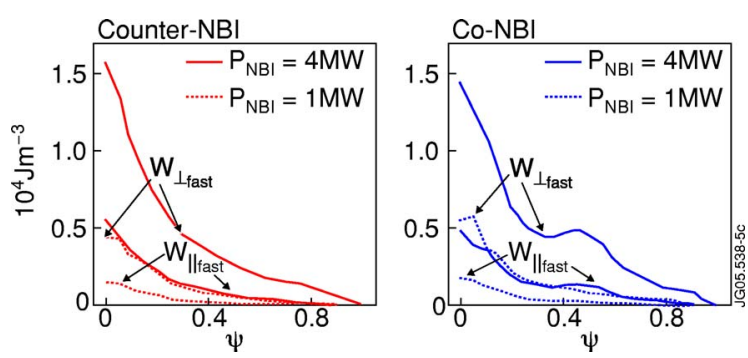

FIG. 6. (Color online) (a) and (b) Profiles of fast-ion energy density $W_{\| \text {ffast }}$ and $W_{\perp \text { fast }}$ calculated with the PENCIL code (finite orbit width effects neglected). 


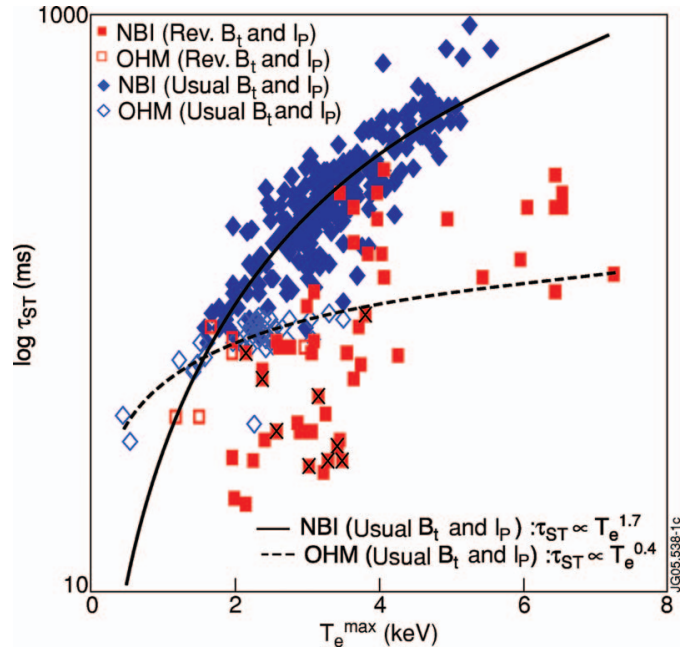

FIG. 7. (Color) Sawtooth period vs maximum $T_{e}$ from a large sawtooth database (many configurations) comparing the usual $B_{T}$ and $I_{p}$ polarity (co$\mathrm{NBI}$ ) in blue and the reversed $B_{T}$ and $I_{p}$ (counter-NBI) in red. The counterNBI power-scan discharges (shown in Fig. 4) are indicated by a black cross inside a red symbol. The open symbols are for Ohmic heating.

is much lower for small or reversed toroidal rotation. ${ }^{14}$ This effect of rotation coupled with a reduction in the stabilizing contribution from fast NBI ions (essentially due to the outward pinch of trapped counterinjected NBI ions and the efficiency of heating to the electrons in the core will be reported in more detail elsewhere). Another factor which could influence sawtooth stability is the build up of impurities for reverse $B_{T}$ plasmas. However, at least for the discharges shown here, the comparison of soft x-ray (SXR) emission does not clearly indicate a significant enhancement of impurities in the plasma core for counter-NBI (see Fig. 2).

In the dedicated sawtooth experiments described above, a direct comparison of sawtooth observations in the usual and reversed $B_{T}$ configurations was only possible for the low-confinement mode ( $L$ mode) and early high-confinement mode $(H$ mode $)$ phases $\left(P_{\mathrm{NBI}}<8 \mathrm{MW}\right)$. Although the power threshold for $L-H$ transition was similar, ${ }^{15}$ the edge-localized mode (ELM) characteristics were different. A higher type III-type I ELM threshold was found for reversed $B_{T}$ and $I_{p}$. The different pedestal conditions led to different core plasma parameters making the comparison of pulses with the same input power more difficult. In order to extend the comparison of sawtooth behavior to higher-power $H$-mode plasmas, data from a wide variety of experiments for edge pedestal and ELM characterization with NBI powers up to $14 \mathrm{MW}$ have also been analyzed. Sawtooth periods obtained with reversed $B_{T}$ and $I_{p}$ were compared with the sawtooth scaling obtained for NBI heating with the usual $B_{T}$ and $I_{p}$ using an existing JET sawtooth database. A plot of the observed sawtooth periods versus the central electron temperature is shown in Fig. 7. For the co-NBI cases the sawtooth period scales with $T_{e}^{1.7}$. For the power scan discussed previously, the sawtooth period decreases with temperature, passing through a minimum at around $3 \mathrm{keV}$. The data shown in Fig. 7 confirm that saw- tooth periods are generally smaller with counter-NBI for similar central $T_{e}$. Thus tokamak operation with counter-NBI is an effective way to reduce the sawtooth period in $\mathrm{H}$-mode as well as in $L$-mode plasmas.

In summary, sawtooth periods are reduced when JET is operated with reversed $B_{T}$ and $I_{p}$, i.e., with counter-NBI. A power scan at constant density showed that with co-NBI the sawtooth period increased monotonically with power, while with counter-NBI the sawtooth period reduced to a minimum at $4 \mathrm{MW}$. A similar trend was observed when comparing the sawtooth period with the toroidal plasma rotation, i.e., with co-NBI the sawtooth period increased with core plasma rotation and with counter-NBI it had a minimum at $f_{\text {rot }}$ $\sim 3 \mathrm{kHz}$. Modifications to the core $q$ profile due to NBCD effects appear to be insignificant. The sawtooth period was shorter with counter-NBI when compared with similar coNBI discharges for both perpendicular and tangential injections. Sawteeth smaller than Ohmic sawteeth were observed for counter- $P_{\mathrm{NBI}}<12 \mathrm{MW}$ in discharges with deeper penetration of heating to the core, which occurs more readily with perpendicular NBI. Tokamak operation with reversed $B_{T}$ and counter-NBI could prove to be a useful tool for reducing the sawtooth period in $H$-mode as well as in $L$-mode plasmas. It should be further explored in the context of avoiding triggering neoclassical tearing modes at low beta.

The work of M.F.F.N. has been carried out within the framework of the Contract of Association between the European Atomic Energy Community and Instituto Superior Técnico (IST) and the contract of Associated Laboratory between Fundação para a Ciência e Tecnologia (FCT) and IST. S.C. and J.G. acknowledge the financial support of the Swiss National Science Foundation.

${ }^{1}$ M. Huguet, K. Dietz, J. L. Hemmerich, and J. R. Last, Fusion Technol. 11, 43 (1987).

${ }^{2}$ E. Westerhof, O. Sauter, M. L. Mayoral et al., Nucl. Fusion 42, 1324 (2002).

${ }^{3}$ M. F. F. Nave, J. Rapp, T. Bolzonella et al., Nucl. Fusion 43, 1204 (2003).

${ }^{4}$ M. J. Mantsinen, S. Sharapov, B. Alper, A. Gondhalekar, and D. C. McDonald, Plasma Phys. Controlled Fusion 42, 1291 (2000).

${ }^{5}$ A. Edwards, A. Bickley, R. Buechse, C. D. Challis, R. Koenig, F. B. Marcus, J. O'Rourke, and D. Storke, Proceedings of 19th EPS Conference on Controlled Fusion and Plasma Physics, Innsbruck, 29 June-3 July 1992, edited by W. Freysinger (EPS, 1992), Vol. 16C, Part I, p. 379.

${ }^{6}$ G. Duesing, H. Altmann, H. Falter, A. Goede, R. Haange, R. S. Hemsworth, P. Kupschus, D. Stork, and E. Thompson, Fusion Technol. 11, 163 (1987).

${ }^{7}$ R. C. Isler, Plasma Phys. Controlled Fusion 36, 171 (1994).

${ }^{8}$ O. Neubauer, G. Czymek, B. Giesen, P. W. Huttemann, M. Sauer, W. Schalt, and J. Schruff, Fusion Sci. Technol. 47, 76 (2005).

${ }^{9}$ H. R. Koslowski, Fusion Sci. Technol. 47, 263 (2004).

${ }^{10}$ D. Bailey, JET Report No. JET-R04, 1998 (unpublished).

${ }^{11}$ F. Porcelli, D. Boucher, and M. N. Rosenbluth, Plasma Phys. Controlled Fusion 38, 2163 (1996).

${ }^{12}$ C. Angioni, A. Pochelon, N. N. Gorelenkov et al., Plasma Phys. Controlled Fusion 44, 205 (2002).

${ }^{13}$ J. P. Graves, Phys. Rev. Lett. 92, 185003 (2004).

${ }^{14}$ J. P. Graves, R. J. Hastie, and K. I. Hopcraft, Plasma Phys. Controlled Fusion 42, 1049 (2000).

${ }^{15}$ Y. Andrew, N. C. Hawkes, M. G. O'Mullane et al., Plasma Phys. Controlled Fusion 46, 337 (2004). 